\title{
The Physics of Intelligence
}

\author{
E. E. Escultura ${ }^{1,2}$ \\ ${ }^{1}$ Institute of Mathematical Sciences and Physics, University of the Philippines Los Baños, College, Laguna, \\ Philippines \\ ${ }^{2}$ International Federation of Nonlinear Analysts, Melbourne, USA \\ Correspondence: E. E. Escultura, 6/13 Sedgwick St., Leumeuah, NSW 2560, Australia. E-mail: \\ escultur36@gmail.com
}

Received: April 17, 2012

Accepted: May 15, 2012

Online Published: August 27, 2012

doi:10.5539/jel.v1n2p51

URL: http://dx.doi.org/10.5539/jel.v1n2p51

The research was funded by Professor V. Lakshmikantham - GVP Institute for Advanced Studies, GVP College of Engineering, JNT University Kakinada, Madurawada, Visakhapatnam, AP, India.

\begin{abstract}
This paper explores the physics of intelligence and provides an overview of what happens in the brain when a person is engaged in mental activity that we classify under thought or intelligence. It traces the formation of a concept starting with reception of visible or detectable signals from the real world by and external to the sense organs, their conversion and encoding on basic cosmic waves as brain waves, transmittal to the creative-integrative region (CIR) of the cortex, storage of sensation components in the respective sensation regions as memory and recall by and recomposition in the CIR as physical concept at its primitive nodal region. It explains formation of simple and complex concept, construction of mathematical space and physical theory and the underlying physical processes involved at every phase starting with reception of external signals by the sense organs. It also explains creation of abstract concepts, i.e., concepts not based on sensation such as mathematical concepts. These activity and capability of the CIR are creative. All of it is accomplished through the new methodology of qualitative mathematics and modeling.
\end{abstract}

Keywords: consciousness, convertor, memory, reflex, receptor, resonance, brain wave, neural network, neural path, nodal region, sensation region, creative-integrative region, genetic activation and encoding

\section{Introduction}

\subsection{The Problem}

The aim of natural science is to know how nature works; that knowledge allows applied scientists and engineers to control nature and serve human needs, e.g., invention of useful technology. Knowledge is expressed or articulated by a physical theory built on natural laws that explain natural phenomena (appearances of nature). While science was content with the conventional methodology of quantitative modeling that describes the appearances of nature mathematically it left long standing problems unsolved, e.g., the 200-year-old gravitational n-body and turbulence problems (Escultura, 1997; Turbulence, 2001) and fundamental questions unanswered, e.g., what the basic constituent of matter and structure of the electron are. The remedy for this inadequacy is the new methodology of qualitative mathematics and modeling that explains nature in terms of its laws introduced in and the main contribution of the author's Ph.D. thesis (Escultura, 1970) and applied to physics for the first time to solve the n-body problem and discover the superstring, basic constituent of matter, required for the solution (Escultura, 1997). The solution required the discovery of the initial natural laws of the grand unified theory (GUT), the foundation of this paper (Escultura, GUT, 2008).

Our problem here is to explain how the brain works, specifically, what happens in the brain when a person is engaged in mental activity that we term thought or intelligence including sensation and instinct. The explanation is provided by the new physics articulated by GUT. GUT itself was developed through qualitative mathematics and modeling. This paper is pioneering and there is no other study on the subject using this new methodology.

The primary hypotheses of this study are natural laws including biological laws upon which GUT and the unified theory of evolution are built (Escultura, Evolution, 2011). The secondary hypotheses are the laws of thought 
(Escultura, Intelligence, 2011). This paper is a physical theory that explains how the brain works. Even in its earlier phase of development the paper gave rise to pioneering work on intelligence (Escultura, Intelligence, 2011; Intelligence and Evolution, 2003; Origin and Evolution, 2010) and mathematics education (Escultura, Creative, 2012) and serves as theoretical foundations for mathematics education (Escultura, Creative, 2012). The paper itself opens up a new field, physical psychology, based on physics through qualitative mathematics and modeling. It also provides the basis for appropriate therapy to counter the effect of the normal shrinking of the brain that comes with aging. A practical application of this theory to medicine along with GUT is the design of appropriate technology for the treatment of genetic diseases (Escultura, Electromagnetic, 2012).

\subsection{Importance of the Problem}

Every pioneering work opens up a new field or more. The application of qualitative mathematics resolved the 360-year-old Fermat's conjecture popularly known as Fermat's last theorem (Escultura, 1998), proved the 250-year-old Goldbach's conjecture (Escultura, New Mathematics and Physics, 2003; Davies and Hersch, 1981) and solved the 200-year-old gravitational n-body problem (Escultura, 1997) that led to the development of the new real number system (Escultura, New Reals, 2009) and the complex vector plane (Escultura, Mathematics of GUT, 2009) through qualitative mathematics. So did the solution of the n-body problem (Escultura, 1997) that led to the development of GUT (Escultura, GUT, 2008) and the unified theory of evolution (Escultura, Evolution, 2011) as theoretical application of GUT through qualitative mathematics and modeling. Similar applications led to the development of the theory of chaos and turbulence (Escultura, Dynamic Modeling, 2004; Chaos and Turbulence, 2011). The ideas in this paper led to a proposed program for creative mathematics education (Escultura, Creative, 2012) and practical application to engineering and medicine in the latter eelectromagnetic treatment of genetic disesases without side effect (Escultura, Electromagnetic, 2012).

\subsection{Relevant Scholarship}

All previous works by the author from 1997 onwards are pioneering because they were developed using this new methodology. The other cited references by other authors provide factual information and experimental data.

\subsection{The Hypotheses and Their Correspondence to Research Design}

The relevant natural laws snd physical principles as hypotheses will be stated as needed. They will be used to build the Physics of Intelligence that explains the nature of thought.

\section{Method}

As already noted, quantitative methods (involving computation and measurement alone) have limited usefulness in mathematics and natural science. It was the critique-rectification of foundations and the real number system (both of which were found to be flawed and the latter inconsistent) using qualitative mathematics that led to the construction of the consistent new real number system (Escultura, The New Reals, 2009); so did the critique-rectification of the complex number system that led to the construction of the complex vector plane (Escultura, 1998; Mathematics of GUT, 2009). This is the reason for the shift to this new methodology that has to its credit over 50 publications (Escultura, Abstracts and Summary of Publications, http://users.tpg.com.au/pidro/) and the only ones in the literature based on this new methodology. Quantitative methods are even more inadequate in the behavioral and social sciences because human behavior and social phenomena are quite complex and not always amenable to computation and measurement. They need more qualitative mathematics and modeling. To-date there are only three other known publications in the behavioral and social sciences based on this new methodology (Escultura, By-passing chaos, 2001; Complex Systems, 2010; Creative, 2012).

This paper is a physical theory built on GUT and the unified theory of evolution through qualitative mathematics and modeling. It is theoretical not statistical research; the latter is appropriate only for conventional quantitative modeling that describes the appearances of thought that this new methodology explains. Therefore, we proceed to build the Physics of Intelligence through scientific and mathematical reasoning based on natural laws called rational thought through qualitative mathematics and modeling.

\section{The Thrust of the Study}

We focus on thought, the secondary function of the brain centered in the cortex. It is also one of the two components of what we call mind; the other component comprised of feelings and emotions centered in the heart. Intelligence is the total conscious capability of thought that includes learning, memory, rational thought and its qualitative model (qualitative mathematics), creativity and critical thinking, the last two items being the core values of science and mathematics, and capability acquired through formal training, self-training, research, experience and study, e.g., mental and athletic skills. Learning, creativity and critical thinking allow the mind to 
form or imbibe values that are particularly appropriate for science and mathematics. The primary function of the brain is control of body processes and movements including the immune system. Doing mathematical and scientific research and constructing mathematical space and physical theory are creative.

We monitor what happens to input from sense organs and its transmittal to and encoding in the creative integrative region (CIR) of the cortex for composition as concepts, storage of its components in the respective sensation regions as memory, their recall to and recomposition in the CIR as concepts and integration into a physical theory. All of it is accomplished through qualitative mathematics and modeling. Intelligence is not possessed by human beings alone. Plant and animal species possess it also at varying levels of complexity and sophistication (Escultura, Evolution, 2011; Intelligence and Evolution, 2003), from the string bean that winds around, chokes and kills the bitter melon to the bee that builds its dwelling in perfect architectural design that conforms to optimization principles (Escultura, Evolution, 2011; Origin and Evolution, 2010).

\section{Mathematical and Physical Concepts}

Mathematical concepts and principles, being man-made and having no physical referents, are formed through formal training, self-training, rational thought and creativity. Physical concepts and natural laws which have physical referents or are based on sensation are also created created in the same manner but also through experimentation, observation and experience, direct or indirect. Rational thought is reasoning based on basic premises or axioms; qualitative mathematics is its qualitative model. Through rational thought we build a mathematical space on its axioms. A physical theory is a mathematical space whose axioms are laws of nature. It explains natural phenomena, processes and transformation of nature and its conversion from one form to another or from one fundamental state to another.

Visible or ordinary matter, one of the two fundamental states of matter, e.g., electron and star, are directly observable but dark matter, the other fundamental state, is only indirectly observable and known only by its impact on visible matter (Escultura, Qualitative modeling, 2009). The full structures of the atom and star were established in 2008 and 2009 (Escultura, GUT, 2008; Mathematics of GUT, 2009; Qualitative Model, 2009). Their formation, evolution and destiny were fully elaborated in 2011 (Escultura, Quantum Gravity, 2011; Macro Gravity, 2011). Mathematical concepts, e.g., distance and time, are also creations of the mind to describe relationship between physical objects; they are not physical objects. For our purposes, this distinction and clarification are important since all physical and chemical characteristics of the human body including physiological changes are physical concepts determined by the genes (Escultura, Evolution, 2011).

An important physical concept is the superstring, basic constituent of matter that fills up everything in the Cosmos (Escultura, Mathematics of GUT); its external configuration is a closed circular helix (figures in Escultura, Quantum Gravity, 2011; From Macro, 2001), its internal configuration a nested generalized physical fractal (Escultura, Generalized Fractals; Edgar, 1990), i.e., the helix contains a superstring traveling through its cycles uniformly at $7 \times 10^{22} \mathrm{~cm} / \mathrm{sec}$ (Atsukovsky, 1990) called toroidal flux; this toroidal flux contains a toroidal flux, a superstring traveling at the same speed through its cycles, etc. Thus, we have here an infinite sequence of superstrings each term except the first a superstring contained in the preceding term and traveling through its cycles uniformly at $7 \times 10^{22} \mathrm{~cm} / \mathrm{sec}$. It is this structure of the superstring called nested generalized physical fractal that makes it indestructible and insures the stability of our universe (Escultura, Mathematics of GUT, 2009). The toroidal flux speed originally measured for the proton (Atsukovsky, 1990) is a constant of nature and applies to the electric current that travels through the conductor at this speed on account of the synchronization and quantization principles (Escultura, Quantum Gravity, 2011).

A superstring is dark if its cycle length (CL) is less than $10^{-14}$ meters, non-agitated if CL $<10^{-16}$ meters and semi-agitated if $10^{-16}<\mathrm{CL}<10^{-14}$ meters. Then we introduce a third phase: agitated or visible, i.e., a segment has CL $>10^{-14}$ meters. Here, superstring refers interchangeably to the fractal superstring itself or the first term of its fractal sequence. Left alone without agitation a superstring shrinks steadily for it shortens the helical path of the toroidal flux and minimizes energy dissipation (energy conservation), its tail end becoming infinitesimal physical continuum (Escultura, New Real Numbers; 2009, Mathematics of GUT, 2009). Non-agitated and semi-agitated superstrings fill up dark matter. Suitably agitated, e.g., by cosmic wave, a segment of semi-agitated superstring bulges to a primum (CL $10^{-14}$ meters), unit of visible matter, e.g., electron, charge $-1\left(1.6 \times 10^{-19}\right.$ coulombs (Encarta)); +quark, charge $+2 / 3$ and -quark, charge $-1 / 3$ (Gerlovin, 1990). The electron, + quark and -quark are called basic prima for they make up every atom. The proton and neutron are suitably coupled basic prima (figures in Escultura, Quantum Gravity, 2011; From Macro, 2001).

We state two natural laws that govern primal interaction, particularly, formation of the atom. The first one was crucial for the solution of the turbulence problem (Escultura, Turbulence, 2001). (The atom, star and galaxy are 
turbulence since they are vortex fluxes of superstrings; called gravitational fluxes in the case of star and galaxy)

Flux-Low-Pressure Complementarity). Low pressure sucks matter and the initial chaotic rush of dark matter towards a region of low pressure stabilizes into local or global coherent flux; conversely, coherent flux induces low pressure around it.

Flux Compatibility. Two prima of opposite toroidal flux spins attract at their equators but repel at their poles; otherwise, they repel at their equators but attract at their poles. Two prima of same toroidal flux spin connect equatorially only through a primum of opposite toroidal flux spin between them called connector.

\section{Qualitative Modeling: A New Methodology}

While psychology can describe how the mind works based on conventional methods this new methodology explains the underlying physical processes involved in terms of natural laws (Escultura, Evolution, 2011; Electromagnetic, 2012), its main tool qualitative mathematics. Qualitative mathematics includes this daily mental activity of the mathematician or scientist:

Making conclusions, visualizing, abstracting, thought experimenting, learning, doing creative activity, intuition, imagination and trial and error to sift out what is appropriate, negating what is known to gain insights into the unknown, altering premises to draw out new conclusions, thinking backwards, finding premises for a mathematical space, searching for the laws of nature and devising techniques that yield results.

The validity of a physical theory, i.e., qualitative model of nature, is tested by this question: is it able to explain its appearances, motion, processes, etc., in terms of natural laws? If the answer is yes, then it is a correct model. A physical theory as articulation of scientific knowledge is distinct from hodgepodge of scientific information. It is the form by which we articulate, store, apply and utilize scientific knowledge. In this regard, we modify our criteria for verification of physical theory:

We take as primary criterion for theoretical validity of physical theory its ability to explain natural phenomena and, secondarily, invention of technology that works since the design is based on prediction of how it will work or function. More precisely, the secondary verification of a physical theory is prediction of an event that actually happens or constant of nature in the theory that is verified or invention of technology that works.

\section{Basic Cosmic or Electromagnetic Waves}

Every piece of matter including living cell vibrates (this accounts for its kinetic energy and temperature) due to the impact of cosmic waves coming from all directions in the Cosmos, its vibration characteristics determined principally by internal structure in accordance with the Internal-External Dichotomy law (Escultura, GUT, 2008). The normal vibration of the atomic nuclei generates basic cosmic or electromagnetic waves propagated across dark matter in all directions. Non- or semi-agitated superstring has only dark or latent energy due to the motion of its toroidal fluxes (energy is motion of matter) but only the toroidal flux of the first term of its fractal sequence is convertible to visible or kinetic energy through suitable agitation by cosmic waves; this sheds light on the indestructibility of the superstring. Basic cosmic or electromagnetic waves are the prime mover of our universe. Aside from converting dark to visible matter and dark or latent energy to visible or kinetic energy they trigger thought and when encoded with vibration characteristics of living cells called brain waves serve as the medium of the brain and gene for their functions (Escultura, Evolution, 2011; Electromagnetic, 2012). The main function of the gene is production of tissues of plants and animals in the cellular membrane through the brain waves it emits that convert superstrings to basic prima (Escultura, Evolution, 2011).

Since the nucleus is fractal (Escultura, Qualitative Model, 2009), so are the basic cosmic waves it generates which it endows with high frequency (great energy). Its kinetic energy is $\mathrm{KE}=\mathrm{hf}$, where $\mathrm{h}$ is Planck's constant and $\mathrm{f}$ frequency but its fractal structure endows it with tremendously huge latent energy $(26 \mathrm{joules} / \mathrm{cu}$. $\mathrm{ft}$. according to de Broglie (Nieper, 1984) or $8.8 \times 10^{8} /$ volts/cm according to Seike Jr (Nieper, 1984) or the equivalent of $18 \mathrm{~kg} / \mathrm{cu}$. meter using relativistic conversion (Gerlovin, 1990). Basic cosmic waves are highly energetic; they penetrate great barriers, e.g., one can tune in the radio in a closed room but scattered by metal that accounts for noise interference.

\section{Resonance}

An important natural law governing wave and vibration interaction is the principle of resonance that says: maximum resonance between waves or vibrations occurs when they have the same wave characteristics. This law was discovered in the course of analyzing and explaining the disastrous final flight of the Columbia Space Shuttle (Escultura, Pillars, 2007; Columbia, 2011). It was the basis of the formulation of the resonance and superposition law (Escultura, Evolution, 2011; Electromagnetic, 2012). Resonance between waves or vibration is 
principally determined by frequency or wave length. For example, when a tuning fork of certain frequency is struck its vibration resonates with and vibrates a tuning fork of the same frequency nearby to produce the same pitch. It regulates radio and TV reception and encoding of signals from the sense organs in the CIR. We summarize this as a natural law.

Law of resonance and superposition. Two waves of the same order of magnitude of wavelength or frequency have maximum resonance when they have exactly the same characteristics and the principal determinant of level of resonance is wavelength or frequency; when two waves resonate their wave characteristics superpose on each other and produce composite waves.

When two waves resonate, i.e., have the same order of magnitude of wavelength, they produce composite wave. Wave can vibrate an object if its order of magnitude of wavelength is comparable to the size of the latter (Escultura, Electromagnetic, 2012).

An object in the Cosmos is observed when it bounces light that hits it or is silhouetted against a source of light or causes interference that occurs when its size is at least the same order of magnitude as the finest wavelength of visible light, $10^{-14}$ meters, since at least some wavelength of light is distorted or demolished and registered by the spectroscope. Objects of lesser order of magnitude in size, e.g., semi- or non-agitated superstring, are not observable directly, its existence known only by its impact on visible matter in accordance with energy conservation. Resonance between waves of similar characteristics reinforces each other provided they have the same phase because their energies add up just as fluxes of the same direction do when their speeds are within the same order of magnitude. However, with the same order of magnitude of wavelength but encoded with different wave characteristics, they superpose their characteristics on each other and produce composite waves. This applies to brain waves (Escultura, Evolution, 2011; Electromagnetic, 2012).

\section{The Human Brain and Its Treasure Trove of Activity}

The human brain weighs 2.2 pounds. On top of the upper lobe and folding over and wrapping around the upper lobe just above the ears, is the cortex, 1/4 inch thick and comprised of two billion neurons. It is quite convoluted that it yields effective surface area of 16 square $\mathrm{ft}$ which enhances its capability to perform. A big portion of the cortex from behind the forehead through the top of the upper lobe and tapering down slightly around it is the creative-integrative region (CIR); around the CIR on the left, back and right of the upper lobe are the sensation regions just above the ears. They store concept components as memory. Together, they constitute the central nervous system with the CIR the center of thought or intelligence. A huge mass of neurons lies under the cortex that extends through the spinal column and the network of nerves that reaches the extremities and remotest parts of the body. This is the command center for all automatic motion and processes in the human body including reflexes. The sense organs are the brain's receptor of signals from the real world external to them.

Applying qualitative mathematics and modeling, we assume that the CIR has all neural paths that resonate with brain waves encoded with signals and their vibration characteristics coming from all the sense organs. In other words, under normal conditions, signals from the sense organs are not blocked off anywhere in the CIR for there are neural paths that resonate with them. Color blindness, for example, is an aberration from the norm; it means that color signals are not encoded in the CIR. A neural path is a linear sequence of contiguous neurons; every pair of contiguous neurons in the sequence joins together at their dendrite tips through a synapse. Brain waves encoded with signals ("information") as vibration characteristics coming from different sense organs pass through bundles of neural paths each carrying brain waves of the same vibration characteristics. As the signals jump from one dendrite tip to another belonging to a contiguous pair of neurons of the neural path, it lights up like a radio transmitter. In fact, the dendrite tips send brain waves in all directions across dark matter. The first brain wave of a sequence that traverses a neural path vibrates, activates and encodes it with its vibration characteristics via resonance. The rest of the terms of the sequence follows and resonates with it without altering the encoded characteristics. Only wave sequence of the same wave characteristics will traverse this neural path again. Incoming brain wave sequence with different wave characteristics, i.e., coming from a different sense organ, carried by a bundle of linear neural paths split at a set of contiguous neurons called primitive nodal region and activate and encode separate linear neural paths sticking out of it. By the time the encoding of an event in the external world is completed a tree (neural network) consisting of linear neural paths sticking out of this primitive nodal region is formed. As long as this neural network is vibrated by continuous inflow of signals with the same vibration characteristics the individual feels the sensations or perceives the event. When the tree reverts to residual or normal vibration the network becomes a simple concept but still encoded in the CIR until at some point when its component neural paths vibration characteristics are transferred to their respective sensation regions in due course, usually during sleep, as components of memory. 
A bundle may consist of several neural paths of the same vibration characteristics that raise the intensity of the sensations so that a single event would send in bundles of brain waves through the various sense organs involved. In the case of sweet sensation, for instance, there would be many neural paths encoded with vibration characteristics coming from the many taste buds for sweetness. However, energy conservation requires that when the concept components are transferred to the respective sensation regions only one neural path with the specific vibration characteristic will be stored in the appropriate sensation region.

Consider a simple event - fire cracker explosion. It triggers sight (color, shape and extent), hearing, odor and, perhaps, touch and pressure sensations. Then the neural bundles from the different sense organs involved transmit all the vibration characteristics induced by the event. When they reach the CIR they activate a primitive nodal region and a tree of linear neural paths sticking from it that captures the various sensations caused by the event. Again when the signals stop the network is deactivated, reverts to its residual or normal vibration and the perception stops. Then the tree will follow the same course until the component vibration characteristics are dispersed to the respective sensation regions as components of memory.

The texture and consistency of the cortex (neurons and other chemicals) is jelly-like so that all impressions on it, e.g., vibration characteristics, are preserved. When the neural network's nodal region is activated, all its component neural paths in the sensation regions are recalled and recomposed there their encoded vibration characteristics reproduced. Then the individual recalls the corresponding concept that captures the original event.

A bundle of linear neural paths carrying different vibration characteristics may split at its nodal region singly or in bundles and a bundle that splits from it may split again at a lower nodal region and activate a neural network there. Then the former nodal region is called a higher or prior nodal region relative to the latter and its neural network models a complex concept, i.e., a concept with a component concept. This happens when, for instance, when the fire cracker explosion is quickly followed by secondary explosions as in fireworks.

\section{Intelligence}

Thought or intelligence as we have defined above is centered in the cortex. It is possessed by humans and advanced species, e.g., some primates, sea creatures and bees (Escultura, Origin and Evolution, 2010; Intelligence and Evolution, 2003). There was a feature on TV about a chimp in a cage under a fruit tree. Sticks and thin ropes were thrown in. After some thought, the chimp tied the sticks into a pole and tried to knock the fruit down. It did not succeed but its intelligence was quite clear. We term lower intelligence other capability of the nervous system that allows lower species to respond to the environment properly such as instinct, e.g., avoidance of danger. It is possessed even by plants. For example, if a bitter melon plant is planted first and climbs a trellis and a string bean is planted later near it, the latter will wind around the former, choke and kills it. If they are planted together, the string bean will go for the trellis but the bitter melon will turn away. This is the same mechanism that works in human instinct which is determined by a gene for either. (Escultura, Evolution, 2011; Origin and Evolution, 2010) as all instincts are even in humans (Escultura, Evolution, 2011). The content of the preceding section is part of intelligence.

\subsection{Recollection}

We pursue what happens to the concept components that had been stored in the sensation regions. When a sensation region is hit by suitable basic cosmic waves directly from the Cosmos or indirectly through the appropriate nodal region in the CIR, an encoded neural component vibrates with exactly the same characteristics as when it was activated and encoded; the agitation sends the encoded signals to its nodal region that, in turn, vibrates, agitates and recalls the other components and recomposes them. Then the individual recalls the concept.

How does the mind recall a non-physical concept, i.e., having no physical referent, since it is neither associated with sensation nor induced by an event? Consider the concept of time. It is solely the result of the CIR's creative capability. Time describes relationship between events; it is determined only in association with contiguous events. It is possible that the individual may get confused as to which of two events came first. This is resolved by recalling events contiguous to each of them. Then only events whose nodal regions are connected by neural paths linearly will lie in a time series in which case the confusion is resolved. One may note that thought follows energy conservation in the sense that the flow of concepts proceeds from activated (agitated or higher kinetic energy) to non-activated regions. During sleep the CIR is relatively more agitated (more energetic) than the sensation regions due to its breadth and location and greater frequency of hit by cosmic waves. This is the reason concept components generally transfer to the sensation regions during sleep.

How does one recall the year a song became a hit? This requires association by the CIR with the right contiguous events that induce physical concepts' recall such as one's place of work, friends and associates, etc., at the time. 
Their recollection (activation of their neural network) fixes the year.

Recall and dispersal of concept components to and from its nodal region involve passage of energy from activated to non-activated network and quite analogous to passage of gas or liquid from high to low pressure (high to low energy). This is in accordance with energy conservation. Moreover, passage of brain waves is simultaneous with sparks at gaps between dendrite tips that kick off the propagation off their propagation across dark matter.

While generation and propagation of brain waves are well understood and explained by natural laws their reception is not fully understood. One such unexplained phenomenon is mental telepathy (ESP) where the individual receives information from external source without involvement of any of the sense organs. There is no scientific confirmation of it in humans but there is in dogs reported by Scientific American. There is also scientific basis for it since brain waves is generated in the CIR and propagated across dark matter.

The CIR is never totally de-activated because of continuous agitation by cosmic waves. This is the basis of dreams. Such agitation results in recall of stored components of neural network and recomposition at the nodal regions. However, since the CIR is not fully functioning during sleep, dreams are often incongruous. Moreover, they are culture based because the content of the sensation regions is drawn from one's experience, creativity and rational thought. Consciousness is due to the normal neural vibration in the CIR sustained by the steady impact of cosmic waves. It stops only when the normal functioning of the CIR stops. However, there are known cases when a person has been pronounced dead only to come to life again after minutes or even hours.

\subsection{Creativity}

The primary components of intelligence are creativity and critical thinking. Creativity includes deductive reasoning, ingenuity, rational thought and intuition in the sense that they create or activate new neural connections or concepts. For example, in deductive reasoning a neural bundle from a higher nodal region of the hypothesis to a lower nodal region of the conclusion is activated that, in turn, recalls the concept and activates its neural network there. The choice of the conclusion, i.e., its nodal region is determined by the individual's way of thinking acquired through formal trainng and self-training, experience and study and it need not be valid. For example, until recently mathematicians relied on formal logic for making conclusions. However, it is not valid for the study of a mathematical space since it has nothing to do with its axioms. Thus, the need for sustained critique of existing knowledge to avoid error. This is the essence of critical thinking. Reasoning in a mathematical space called rational thought relies solely on its axioms (Escultura, Electromagnetic, 2012; Creative, 2012).

Creativity is the mind's capability to compose new concepts, simple or complex and activate new neural paths or bundles. Inductive reasoning which is also creative is the reverse: it activates neural bundles from a lower nodal region to a higher nodal region. We can look at a mathematical space as a complex concept since it is made up of concepts and propositions such as its axioms, elements, operations, functions, theorems and their relationships. The same is true of a physical theory except that its axioms are laws of nature. In other words, a physical theory is a mathematical space whose basic premises or axioms are laws of nature.

However, not all created concepts make sense. Take the concept

$$
i=\sqrt{ }(-1)
$$

which is supposedly the root of the equation

$$
x^{2}+1=0
$$

among real numbers. By applying suitable valid binary operations on both sides of equation (1), we obtain,

$$
\mathrm{i}=1 / \mathrm{i}=-\mathrm{i},
$$

from which follows that $\mathrm{I}=0$ and $\mathrm{i}=0$ both of which are contradictions in and collapse both the real and complex number systems to the null space $\{0\}$. The culprit here is the concept $i$ which is vacuous, does not exist, since equation (2) has no solution in the real number system. (For full critique-rectification of the real and complex number systems, see Escultura, New Reals, 2009; Mathematics of GUT, 2011).

The brain is the most active organ of the body; utilizing a disproportionately large amount of the energy from the food we take - $25 \%$ - compared to the ratio of its weight to body weight which is only about $1.6 \%$. Most of it is used for mental activity and control of body functions. This is the reason high technology is expensive; its conceptualization requires high concentration of brain power. Now, GUT technology is just as expensive but its capability is a million times that of high technology (Escultura, Electromagnetic, 2012). 


\subsection{Complex Concepts and Theories}

Through creative activity thought is able to build a pyramidal hierarchy of nodal regions and their neural network that models a mathematical space or physical theory at the apex of which is its central nodal region. All component neural paths from the primitive nodal regions are connected by neural bundles through higher nodal regions that join into a single bundle that connects to the central nodal region. This unification of concepts and principles into a single mathematical space or physical theory allows full play for creativity, critical thinking and applications, theoretical and practical. Moreover, since mathematics is the language of science, a physical theory has necessarily a mathematical component neural network. Of course, autonomous neural network may exist, e.g., hodgepodge of information that have nothing to do with mathematics or science.

\subsection{Reception and Conversion}

A sense organ has two components, receptor of visible signals and convertor of these signals to brain waves for transmission to the CIR through neural bundles. In some sense organs the receptor and convertor are one, in others separate.

Consider the organ of taste, the tongue; its receptor consists of separate groups of taste buds (for sweet, sour, bitter and salty) along its edge determined and differentiated by their vibration characteristics that make them resonate with and encode on basic cosmic waves generated by food molecules of corresponding tastes, in accordance with the resonance and internal-external dichotomy laws (Escultura, GUT, 2008). The most accurate medium for molecular vibration is liquid, saliva in this case. Take a sugar molecule. It must be soaked in saliva so that molecular vibration is transmitted accurately to, resonates with and vibrates the taste buds for sweetness attached to the nerves at their base. Each taste bud for sweetness resonates with and is vibrated by the signals from the sugar molecule so that its vibration characteristics (for sweetness) superpose on or get encoded on the normal nuclear vibration of the nerve fibers (neural paths) at its base and transmitted to the CIR through bundles of neural paths. Upon entry in the CIR the bundles activate a nodal region and compose the appropriate neural network there that models the taste "sweet" including flavor and texture. Thus, its vibration characteristics may have come from different sense organs. In this example, the taste bud is both the receptor and convertor of the visible signals.

Understanding sweet sensation has yielded technology for control of diabetes. Insulin brings sugar inside the cells for nourishment. When pancreatic secretion of it is inadequate, a condition suffered by diabetics, blood sugar level rises. Sugar is viscous and high blood viscosity can damage the fine blood vessels of the kidney and eye, raise blood pressure and cause other complications. Moreover, too much glucose around the cell blocks the passages to and keeps the cell undernourished. That is why diabetics eventually lose muscle tissues.

Since taste is due to the physical structure of food molecule that determines its vibration characteristics it can be simulated by using non-viscose compound. In fact, this is what synthetic foods are. Synthetic sugar "Equal" and "Splendida" consist of suitably designed carbon molecules with the vibration characteristics of sugar molecules; it is $550 \%$ sweeter than ordinary sugar of the same concentration but without harmful effect on the diabetic. Now, there is great number of synthetic foods, e.g., beef and crab "meat" from vegetables which are organic (carbon base), with their taste but without sugar and cholesterol.

Take another sensation - hearing. In this case the convertor is separate from the receptor. The eardrum is receptor of ordinary sound waves. The outer ear catches and directs sound waves through the outer ear canal to the eardrum which vibrate it by resonance. On the other side of the eardrum is the inner ear consisting of the cochlea, a spiral canal filled with liquid (again, an accurate medium for vibration). Near the eardrum immersed in fluid are the three tiniest bones in the body: malleus (hammer, one end - handle - connected to the inner side of the eardrum), incus (anvil, under the other end of the hammer) and stapes (stirrups, floating on the liguid as stabilizer and amplifier), in triangular arrangement that allows detection of where the sound is coming from. Strands of hair sticking out of the nerves grouped according to vibration characteristics line the interior of the cochlea. They are the convertor of sound waves to brain waves for transmission to and composition as concepts in the CIR through neural bundles. Sound waves from external sources vibrate the eardrum. As the eardrum vibrates, the hammer hits and vibrates the incus and the stapes near it. The stapes stirs the cochleal fluid, stabilizes the vibration and propagates it. The latter resonates with and vibrates the corresponding strands of hair that line the inner wall of the cochlea via resonance. Their atomic nuclei encode the vibration characteristics on basic cosmic waves turning them to brain waves that carry and transmit information to the CIR through neural bundles (the nerves) for activating a corresponding neural network. In this case, the hair strands are the convertor. Energy conservation insures that when a concept's components are stored in the sensation regions its neural network in the CIR deactivates and restores normal vibration and vice versa. 
Faint signal from a sense organ is amplified by a process called sodium jump: neural cell ionization of sodium atom along the neural path that creates electrical potential followed by its jump to lower potential accompanied by electrical impulse. What causes this ionization, i.e., expulsion of orbital electrons from the neural atoms? The only force that does it is the energetic brain waves passing through. The sodium jump amplifies nerve cell vibration and raises the energy of the carrier brain waves and encoded signals.

\subsection{Rational Thought}

Making conclusion in deductive reasoning amounts to activating a neural path from the nodal region of the premise to the lower nodal region of the conclusion where its components are recalled for recomposition. Part of conscious thought is the ability of the CIR to make a command by vibrating a nodal region suitably and selecting a neural path to agitate a particular lower neural region or agitate the neural network of the conclusion. This capability is verified by recent findings that the mind can turn on a prosthetic arm or direct the cursor to hit an icon. The choice of the neural path to the conclusion comes from training, peer pressure, experience, critical thinking and analysis. That is how one's way of thinking is formed and consolidated as value.

In inductive reasoning a neural path to a higher nodal region is activated where a component of a neural network there is an inference. In contrast to deductive reasoning where the conclusion is a necessity inference is not; it is simply a correspondence. Both deductive and inductive reasoning are components of rational thought and qualitative mathematics is its model along with creativity and critical thinking. All activities that make up qualitative mathematics belong to intelligence. However, one's intelligence is not inborn but due to formal training, self-training, experience and research. This applies to one's way of thinking.

What happens in scientific and mathematical reasoning? Every field of natural science and mathematics has a central nodal region to which all neural paths of concept components at lower nodal regions are connected by neural bundles down to their primitive nodal regions. In turn, the central nodal regions of the various fields of science and mathematics are connected to each other through a super nodal region. This allows the CIR to activate specific parts of any branch of science as needed. For instance, a physicist doing research may activate a specific neural network of mathematics through its central nodal region as needed. Thought, particularly research raises the level of neural network interconnections and deepens and enhances grasp of the subject matter. In general, mental activity enhances neural interconnections that raise the capability of the brain described as wisdom. Sustained mental activity, particularly, rational thought, is the antidote to the normal narrowing of the brain (due to neural deterioration resulting in neural spaces detected through MRI) that comes with aging that could lead to senility or even dementia. Enhanced neural interconnection retains the normal functions of the brain, particular, its primary function of control over body processes that a person who remains mentally creative enjoys relatively good mental and physical health in their $80 \mathrm{~s}$ and $90 \mathrm{~s}$. This is supported by scientific study. In particular, active mathematicians are known to be mentally active in their eighties and 90s. Among the notable ones in this group were Bertrand Russell (96), John Littlewood (96) and L. C. Young (95), who wrote papers in his 90 s.

Short of a physical theory a discipline of science is a hodgepodge of disparate information and experimental data and even with a physical theory in each discipline but short of a unifying theory like GUT human knowledge is a disparate cluster of specialized knowledge. GUT, however, binds it together into a unified physical theory at a central super nodal region.

\subsection{Value, Perception and Cognition}

The CIR can recall and recompose concept components at their respective nodal regions. Psychology says ones' perception of event comes mainly from memory, only $20 \%$ from events outside. Psychologists use this knowledge to assess one's personality by asking the subject to tell what he perceives from a set of objects. It reveals the contours of one's life experience.

Values including one's way of drawing out conclusions are learned in accordance with Pavlov's theory of learning or conditioning. They consist of a system of "correct" choices, decisions, conclusions, etc., corresponding to the right neural connections to their respective concepts in the CIR. They are formed principally through training, peer pressure, experience and study. In this sense, one's way of thinking belongs to the category value. Correct choices are imbibed by approval and incorrect ones by rejection first by parents and, later by teachers, peers and experience. Correct choices, decisions, etc., are encoded as neural connections to their premises, principles or convictions modeled by neural network in the CIR. A discipline of knowledge (e.g., mathematics or physics or biology or political science) consists of a system of concepts and values (including rules of inference) encoded as suitable neural network whose nodal regions are connected to the central neural region of the discipline at which its axioms or natural laws (of physical theory) or social principles are composed. 
Different disciplines of knowledge may be autonomous but connected through their central nodal regions to a super central nodal region so that a researcher can switch focus from one discipline to another through the latter and down through their central neural regions. A normal individual may have several connected systems of knowledge including practical knowledge that allows him to function day-to-day. Scientific knowledge, distinct from hodgepodge of information, is qualitatively modeled by physical theory, the form we articulate, preserve and apply.

In rare cases distinct and disconnected systems of values are involved and the individual suffers from multi-personality problem where one personality may or may not know the others. Sometimes systems of values are incoherent or contradictory that may lead to total mental and physical collapse such as epileptic seizure.

How does intelligence work in practical affairs? During, say, a game of bowling the player models the lanes at a central nodal region by appropriate neural network where the nodal region of the bowling lane is the focus. The player commands the body to play the game on the basis of his perception of the lane, knowledge of the game and previous experience. A skillful bowler accurately models the lane on the basis of which the CIR commands precise execution.

In any physical endeavor thought gives command through brain waves that activate appropriate parts of the body to execute it. Sometimes thought triggers automatic execution. For instance, when one thinks of lemon brain waves agitate and convert superstrings to saliva in the salivary gland.

In theoretical research thought utilizes knowledge, observation, experimental data, training, social or physical theory, if any, and mathematical tools to "discover" and formulate (create) laws of nature upon which to build a physical theory. Although natural laws refer to physical concepts their statements and articulation are mathematical principles because they have no physical referents, i.e., one does not find them in the external world. They are created and articulated by thought in the appropriate language based on synthesis or analysis of known information, observation and experimental data.

For the main function of the brain as control center of all processes in the body, stress dulls its ability to keep the auto-immune system in top shape that the latter is unable to do, among others, its normal function of destroying unwanted systems. Ordinarily, the body has tolerable level of cancer cells and the auto-immune system adequately maintains it by destroying some of them when needed. However, under stress, it may fail and be overcome by their replication; then the individual becomes sick. That is why healthy body and mind must go together. In some cases extreme stress or emotional trauma may cause physiological imbalance such as raised level of body regulators, e.g., serotonin. This is what happens in depression where the neural network vibrates even when they are not supposed to and the individual hears voices or sees things (visual or audio hallucination) that are non-existent, or makes unusual decision, e.g., attempting suicide. Such imbalance may be due to a gene created by emotional trauma; in the latter physiological change may occur that is eventually encoded genetically in accordance with the law of genetic encoding (Escultura, Evolution, 2011). In general, strong emotion is accompanied by physiological changes, e.g., raised adrenalin secretion associated with anger. Strong sensation may also cause physiological changes, e.g., extreme pain produces molecule in the neural membrane (Krylov, 1999, Osipenko, 1990). Physical processes in the brain involved in mental activity have been quantitatively modeled (Pokrovsky, 1986, 1989, 1999).

When the CIR lacks the ability to direct or control cosmic waves the person suffers from a condition called autism that erodes the ability to concentrate or focus. In one form the person is simultaneously aware of everything around him and cannot respond properly. Naturally, he has short attention span since other sensations compete for attention and becomes hyperactive (therapy can now control this condition).

\section{Learning}

Learning is not only fitting new concepts, information, etc., into knowledge in one's thought but also storing their components in the appropriate sensation regions; moreover, they can be recalled and recomposed. If the latter cannot be done, nothing is learned. A normal mind is both selective and focused. It entertains only very few signals from the external world. For example, a student is aware of what happen in the classroom but unaware of the noise outside. Only a few of the former is registered and still fewer is retained as long-term memory. What is the basis of long-term memory? It is resonance in the broad sense, i.e., new information is learned or retained when it fits a knowledge system in thought based on qualitative modeling or values particular to it. This process involves neural network encoding, an active mental activity requiring concentration.

Moreover, information that requires deep concentration to understand and integrate into a knowledge system is never forgotten once grasped. This is due to the vastness of neural interconnections that comes with it. In turn, 
this enhances recollection of knowledge. One technique for enhancing retention is listening to and concentrating on the lecture rather than taking note which can be distracting. This way when the student grasps the subject thoroughly and deeply, inducing extensive neural interconnections, it eases recollection. (A concept that is not recalled is not learned). This is probably the reason a student who went through great adversity that keeps him busy with many things and has little time to review his notes strives to grasp the lectures instead and turns out to be an achiever in his fields. Another learning enhancer is listening to the lecture critically by raising question in his thought whether what is being said makes sense. Of course, another avenue for learning is creativity which happens in research.

What facilitates learning? One is critical thinking that gives rise to its offspring - creativity. Taking a critical look at existing knowledge, thought identifies weakness that triggers search for remedy. It was this critical attitude that triggered critique-resolution of the foundations of mathematics and the real and complex number systems that paved the way for the resolution of Fermat's last theorem (FLT) in 1998 (Escultura, 1998) and construction of the new real number system and complex vector plane (Escultura, Mathematics of GUT, 2009). Moreover, critical thinking enhances mental activity and expands neural interconnections the effect of which is to deepen and broaden grasp of the subject matter that enhances retention and retrieval of information. In physics critique of its foundations revealed inadequacy of quantitative modeling. The remedy was qualitative mathematics and modeling that gave rise to theoretical physics - GUT - where there was only mathematical physics previously.

\section{Learning Principles}

We identify learning principles with practical applications for mathematics-science education. We first summarize the discussion of Subsection 9.6 as learning principles.

Learning-efficiency-memory principle. Learning efficiency is in direct proportion to the level of memory in thought.

Principle of complexity and retention. Learning effectiveness increases with the level of complexity of the subject matter and mental processing required; the more complex the subject matter the higher the level of concentration needed to understand it and the more permanent the retention.

One's ability to concentrate is enhanced by serious thought in the midst of great distraction. This was done by the tennis champion Agassi. The author grew up next door to the power plant and before the computer became a household item drafted his papers in noisy coffee shops instead of the deafening silence of the study room. All of these raised the power of concentration.

The rate of learning graph as a function of time approximates the logarithmic curve that tapers off away from the origin. This is familiar to psychologists. The tapering off reflects the law of diminishing return as the brain gets tired, thought loses interest and concentration drops to zero. When learning requires high level of concentration due to the complexity of the subject matter the rate of retention graph rises towards a vertical asymptote at the completion of the learning process signifying permanent retention or memory. This is true of complicated scientific analysis or principle. When learning involves only mechanical activity, e.g., card concentration game, the curve drops off to zero when the activity stops.

Learning and resonance principles and recollection. Thought only retains information that makes sense, i.e., fits some knowledge in it and, in mathematics or science, processes only information that fits some mathematical space or physical theory.

There are, of course exceptions, like trivia, where there is motivation to win some prize at TV competition. Retention is also enhanced by the number of sensations involved in the course of learning something. Thus, the rationale for multi-media teaching aids involving sight, hearing and touch (hands-on).

Learning is not a creative process unless it is the result of original research. Creativity, e.g., obtaining results from frontier research, comes from extensive experience and study and the right orientation. For instance, research creativity is limited in fields already well explored by others.

Retrieval of information or recollection is not learning but looking for information whose concept components are already encoded in the sensation regions. However, it can increase one's receptiveness to related incoming information and enhance learning resonance. In some cases the incoming information triggers recollection.

Absent-mindedness is due to low level of concentration at the time of encoding. For instance, one picks up an umbrella as a reflex action to rain; consequently, he is likely to leave it in the bus when the rain stops.

The material basis for recollection is agitation of the neural network that stores the components of information. Then they are recalled and recomposed at their nodal regions. Activation of a sensation component of a concept 
activates the other components through their common nodal region. Contiguous events are encoded neural network with common interconnected nodal regions.

Well-grasped complicated topics are easy to recall due to the enormity of concepts and, therefore, interconnected network involved. Activation of one network triggers activation of others connected to it.

Principle of association and neural network interconnection. The more interconnected and contiguous the encoded neural network is the easier and more precise the recollection.

Learning occurs not only through cognition of similarity and regularity of patterns but also through comparison and contrast by drawing out extreme situation and dramatizing the effect or impact of opposite conclusions. Stressing a point and reducing an undesirable conclusion to absurdity are among the practical tactics for effective teaching. They broaden neural bundle activation and enhance permanency of retention. At the same time, reduction to absurdity or contradiction jolts thought into recognition of error and negation of a particular proposition. Their effect is to raise the level of neural interconnection and enhance the ease of recollection and clarity of details. Conversely, concepts not recalled for a long time are likely to be drawn into the subconscious. Physically, this means that when the associated neural network remains un-agitated for a long time, it becomes difficult to agitate and retrieve the information encoded in it. This is analogous to the phenomenon of atrophy. Psychologists have devised techniques for drawing out information from the subconscious or, perhaps, blocking off unpleasant memory. In the former it amounts to increasing contiguous concepts around the information and, in effect, raising the intensity of agitation. In the latter it amounts to altering the recollection of previous events. This is not an unusual phenomenon. In some cases, suitable psychological technique can make an innocent person confess to a crime he did not commit thinking that he did. Constant repetition of a lie may cause it to be perceived as truth. This was used by the protagonists during the Cold War.

Structure and coordination of knowledge. A discipline of knowledge is encoded as system of neural network with interconnected nodal regions connected to a central nodal region. Different disciplines have interconnected central nodal regions, each autonomous but can be activated fully or partially. However, they are all connected to a central super nodal region through which any part of the whole network can be activated.

When the mind is engaged in research, e.g., physics, neural network of related disciplines, e.g., mathematics, also activates when needed through the central super nodal region.

Since neural network is a physical entity encoded with vibration characteristics it is ultimately genetically encoded and when the neurons die the encoded information (vibration characteristics) is passed on to the replacement. Thus, the physical components of the hunting skill of the female lion is encoded in the gene. An example of such physical characteristic is the cosmic intensity threshold for activating a network. Another is the encoding sensitivity of the neurons. Persons with photographic memory have high encoding sensitivity and low degradation of neural vibration characteristics. This information might have implications for the study of intelligence, particularly, learning capability.

The physical components of the hunting skill of the female lion are encoded in the gene. However, the female cub needs training to actualize it. Therefore, mother lion borrows the cub of a prey in the morning for training and returns it to its den in the afternoon to mature and become a prey (Escultura, Origin and Evolution, 2010).

There are rare cases where the neural network interconnections are genetically encoded, not just the physical characteristics of the neurons. An example is Devi Shakuntala's ability to compute huge numbers - up to 13 digits (the modern computer can only compute 11) - and yield the result automatically without her really knowing how she did it. The only possible explanation is there was in her cortex suitable interconnected neural network that got activated and carried out the computation automatically as soon as the numbers were fed in the same way reflex is activated by certain stimuli. Both this special skill and reflex are genetic. After all, the synapses that join the dendrites are determined by a gene. Such exceptional ability is likely to arise in old cultures due to genetic acquisition evolutionary advance. Devi Shakuntala was an Indian and India has the oldest culture (the Ice Age comes at interval of 10,000 years and it came to India Twice (Lakshmikantham, 1999).

\section{Strategy for Research}

Theoretical research is creative. But, as a beginning researcher, a novice, so to speak, e.g., graduate student, one must train in creativity first by starting with the advisor's works. More is needed: taking courses under well accomplished professors in different fields even if they are not required in the program of study. The idea is to know how they established themselves and what their approaches and styles were. Having done that it is necessary to establish one's turf away from their shadows to be able to grow and surpass them in some field if possible. A good advisor trains his student to beat him somewhere (Young, 1980). One may adopt their styles but, 
preferably, develop his own. It is necessary to start with a modest but non-trivial problem just to gain confidence (the author started with the wild oscillation, $\mathrm{f}(\mathrm{x})=\sin 1 / \mathrm{x}$ and tried to figure out what to do with it; this gave birth to the generalized integral for set-valued function since $\mathrm{f}(\mathrm{x})$ is set-valued at the origin (Escultura, Generalized Integral). Once achieved, one may go for the big one. Even here it is prudent to keep away from the veterans in their fields but compete with them on a higher plane, i.e., by creating a new field and reformulating and resolving an old problem there (e.g., the 360-year-old Fermat's last theorem) or formulating a new one and resolving it. The trick is to find a really challenging unsolved problem that even the veterans shy away from. Most researchers jump into such problems and try different tactics to solve it but guided by conventional approaches and methods. Considering that, perhaps, thousands have tried it before but failed, the chance that this time it will be solved without something new to bear on the problem is nil. So where does one go from here? Spend a lot of time figuring out why the problem defies solution. The right answer to this question is the key to the solution.

\section{References}

Escultura, E. E. (1997). The solution of the gravitational n-body Problem. J. Nonlinear Analysis, A-Series: Theory, Methods and Applications, 30(8), 5021-5032. http://dx.doi.org/10.1016/S0362-546X(96)00133-2

Escultura, E. E. (2001). Turbulence: theory, verification and applications. J. Nonlinear Analysis, A-Series: Theory: Method and Applications, 47, 5955-5966. http://dx.doi.org/10.1016/S0362-546X(01)00697-6

Escultura, E. E. (1970). The Trajectories, Reachable Set, Minimal Levels and Chains of Trajectories of a Control System. Ph.D. Thesis, University of Wisconsin, in press, LAP LAMBERT Academic Publishing GmbH \& Co., KG.

Escultura, E. E. (2008). The grand unified theory. J. Nonlinear Analysis, A-Series: Theory: Method and Applications, 69(3), 823-831. http://dx.doi.org/10.1016/j.na.2008.02.043

Escultura, E. E. (2011, September). The unified theory of evolution, In E. E. Escultura (Ed.), Scientific Natural Philosophy, Bentham EBooks. Retrieved from http://www.benthamscience. com/eBooks/9781608051786/index.htm

Escultura, E. E. (2012, February). Creative Mathematics Education. Open Access J. Creative Education. Retrieved from http://www.scirp.org/journal/PaperInformation.aspx?paperID=17266

Escultura, E. E. (2012). Electromagnetic Treatment of Genetic Diseases. Open Access J. Biomaterials and Nanobiotechnology. Retrived from www.ccsenet.org (online end of May, 2012).

Escultura, E. E. (2001). By-passing chaos with a theory of turbulence and development, In Perez, D., \& Bonzo, B. (Eds.), Finance Stochastics. World Scientific, London.

Escultura, E. E. (2011). Qualitative modelling for complex systems. J. Problems of Nonlinear Analysis in Engineering Systems, 17(36), 79-85.

Escultura, E. E. (1998). Exact solutions of Fermat's equation (A definitive resolution of Fermat's last theorem, $J$. Nonlinear Studies, 5(2), 227-254.

Davies, P. J., \& Hersch, R. (1988). The Mathematical Experience. Birkhäuser: Boston.

Escultura, E. E. (2003). The new mathematics and physics. J. Applied Mathematics and Computation, 138(1), 145-169.

Escultura, E. E. (2003). The mathematics of the grand unified theory. In E. E. Escultura (Ed.), Scientific Natural Philosophy, Bentham. Ebooks. Retrieved from http://www.benthamscience.com/ebooks/9781608051786/index.htm doi:10.1016/j.na.2008.11.003

Escultura, E. E. (2009). The new real number system and discrete computation and calculus. J. Neural, Parallel and Scientific Computations, 17, 59-84.

Escultura, E. E. (2011, September). The Theory of Chaos and Turbulence. In E. E. Escultura (Ed.), Scientific Natural Philosophy. Bentham Ebooks. Retrieved from http://www.benthamscience.com/ebooks/9781608051786/index.htm

Escultura, E. E. (2003). The theory of intelligence and evolution. Indian J. Pure and Applied Mathematics, 33(1), 111-129.

Escultura, E. E. (2010). The origin and evolution of biological species. J. of the Science of Healing Outcomes, 6, $17-27$. 
Escultura, E. E. (2009). Qualitative model of the atom, its components and origin in the early universe. $J$. Nonlinear Analysis, B-Series: Real World Applications, 11, 29-38. http://dx.doi.org/10.1016/j.nonrwa.2008.10.035

Escultura, E. E. (2011). Quantum Gravity. In: E. E. Escultura (Ed.), Scientific Natural Philosophy (pp. 61-80). Bentham. Retrieved from http://www.benthamscience.com/ebooks/9781608051786/index.htm

Escultura, E. E. (2001). From macro to quantum gravity, J. Problems of Nonlinear Analysis in Engineering Systems. 7(1), 56-78.

Escultura, E. E. (in press). Extended Geometrical and Generalized Fractals, Chaos and Application. In Mark Columbus (Ed.), Fractal Classification. Nova Science Publishers. Retrieved from http://www.novapublishers.com

Edgar, G. A. (1990). Measure, Topology and Fractal Geometry. Springer-Verlag: New York.

Atsukovsky, V. A. (1990). General ether-dynamics; simulation of the matter structures and fields on the basis of the ideas about the gas-like ether. Energoatomizdat: Moscow.Encarta Premium.

Nieper, H. A. (1984). Revolution in Technology, Medicine and Society. Management Interessengemeinschaft für Tachyon-Feld-Energie: Odenburg, FRG.

Gerlovin, I. L. (1990). The Foundations of United Theory of Interactions in a Substance. Energoattomizdat: Leningrad.

Escultura, E. E. (2011, September). Macro Gravity. In E. E. Escultura (Ed.), Scientific Natural Philosophy, Bentham EBooks. Retrieved from http://www.benthamscience.com/ebooks/9781608051786/index.htm

Escultura, E. E. (2001). From macro to quantum gravity. J. Problems of Nonlinear Analysis in Engineering Systems, 7(1), 56-78.

Atsukovsky, V. A. (1990). General ether-dynamics; simulation of the matter structures and fields on the basis of the ideas about the gas-like ether. Energoatomizdat: Moscow.

Escultura, E. E. (2007). The Pillars of the new physics and some updates. Nonlinear Studies, 14(3), 241-260.

Escultura, E. E. (2011, September). Columbia's' Final Flight: Crossroads for Science. In E. E. Escultura (ed.), Scientific Natural Philosophy. Bentham EBooks, Retrieved from http://www.benthamscience.com/ebooks/9781608051786/index x.htm

Lakshmikantham, V. (1999). The Origin of Human Past: Children of Immortal Bliss. Bharatiya, Vidya Bhavan: Bombay.

Young, L. C. (1980). Mathematicians and Their Times. North-Holland: Amsterdam.

Escultura, E. E. (2004). Dynamic Modeling of Chaos and Turbulence. J. Nonlinear Analysis, A-Series: Theory: Method and Applications, 63(5-7), e519-e532. 